\title{
Sistema de medición de la calidad de la Educación Física Chilena: un análisis crítico
}

\author{
Alberto Moreno Doña* \\ Enrique Rivera García** \\ Carmen Trigueros Cervantes ${ }^{* * *}$
}

\begin{abstract}
Resumen: En este trabajo se analiza, cualitativa y críticamente, el sistema de evaluación utilizado por el Ministerio de Educación de Chile para medir la calidad de la educación física como asignatura del currículum escolar. Los procedimientos de análisis empleados, en general se ciñeron a las propuestas de fragmentación y articulación de la "Grounded Theory". Se concluye que dicho sistema no da respuesta a un concepto de calidad previamente explicitado; muestra una importante limitación conceptual en la relación salud y condición física; establece una lógica evaluativa escasamente formativa y genera cierta desvalorización profesional de los docentes.
\end{abstract}

Palabras clave: .Gestión de calidad. curriculum. Medida

\section{INTRODUCCIÓN}

La evaluación siempre ha estado ligada a los procesos de enseñanza y aprendizaje. Si en un principio su objetivo era la búsqueda de la máxima eficacia y la selección de los mejores estudiantes, generalmente desde la valoración de las capacidades cognitivas, a partir de los años noventa del siglo XX comienza a evolucionar hacia un enfoque más preocupado de evaluar la adquisición de competencias complejas y convertirse en instrumento útil de cambio

\footnotetext{
"Escuela de Educación Física. Facultad de Filosofía y Educación. Pontificia Universidad Católica de Valparaíso. Valparaíso, Chile. E-mail: alberto.moreno@ucv.cl

"Facultad de Ciencias de la Educación. Universidad de Granada. España. Granada, E-mail: erivera@ugr.es

"'Facultad de Ciencias de la Educación. Universidad de Granada. Granada, España. E-mail: ctriguer@ugr.es
} 
de los procesos de enseñanza y aprendizaje producidos en contextos concretos. Será a partir del nuevo siglo, en simultaneidad con el triunfo definitivo de las políticas económicas neoliberales, cuando los estados comienzan a ver la necesidad y conveniencia de que la educación utilice indicadores de eficacia (otros llaman calidad) y rendimiento similares a los utilizados en los mercados. Estamos ante el impulso definitivo de los modelos de evaluación externa y comienza la carrera local, regional, nacional o internacional por someter al sistema educativo a pruebas estandarizadas del tipo del Third International Mathematics and Science Study (TIMSS), el Progress in Reading and Literacy Study (PIRLS), o el más conocido en nuestro contexto, el International Programm of Student Assesment (PISA). Todos estos sistemas de evaluación toman como referencia el informe Definition and Selection of Competencies (DeSeCo) (OCDE, 2002), que da el espaldarazo definitivo a lo que venimos llamando 'Enseñanza basada en competencias' o lo que es lo mismo, poner en el centro del proceso de enseñanza y aprendizaje a una forma de evaluación técnica (PÉREZ-GÓMEZ; SOTO 2011; SERVÁN 2011).

¿Qué se pretende desde la evaluación externa? Desde la racionalidad técnica, se trataría básicamente de conocer mejor la realidad educativa sometida a evaluación (MONARCA 2012). Lejos de la declaración de neutralidad que se pretende desvistiendo de toda ideología a la educación y las intenciones que declara de construir conocimiento (PÉREZ; SOTO 2011), lo que se manifiesta claramente es una clara intencionalidad de transmitir una ideología neoliberal oculta que puede tener consecuencias sociales impredecibles. Recordando las palabras de Stake (2006, p.34), no debemos olvidar que "la evaluación es una tecnología destinada a asignar valor a los objetos, los hechos, los procesos y las personas". Frente a la mirada técnica de la evaluación, autores diversos (ESCUDERO 2010; MARTÍNEZ 2011; PÉREZ; SOTO 2011; PERRENOUD 2008), apuestan por una racionalidad hermenéutica que otorgue valor a la compresión de cómo y por qué se producen los procesos, donde conocer la realidad como fenómeno complejo es lo importante, dejando como accesorio el conocimiento producido desde las pruebas estandarizadas. Algunos autores incluso apuestan 
por una evaluación interna (auto-evaluación) del sistema educativo. Simons (2011), ofrece una alternativa interesante desde la implicación de todos los agentes del centro educativo en el proceso desde la realización de Estudio de Casos que faciliten el desarrollo profesional en el centro y la concienciación de la comunidad educativa de cara a exigir los apoyos necesarios a la administración para cambiar desde dentro.

Sin embargo, los actuales modelos de evaluación externos predominantes a nivel internacional, y sustentados desde la racionalidad técnica, están provocando, desde la elaboración de los ranking de excelencia, la creación un sentimiento en los diferentes agentes que intervienen en la escuela, especialmente en el profesorado, de estar siendo empujados, desde la fiscalización y jerarquización a la que se les somete, al abandono de las actitudes de cooperación, tan necesarias para la innovación y mejora escolar, y su sustitución por prácticas de competencia que les permita sobrevivir en el sistema (GOLDSTEIN, 2001).

Los antecedentes de estos modelos de evaluación en Chile, contexto en el que se centra este trabajo, se encuentran en la década de 1990 con la llegada de la democracia. Durante aquella época se comenzó a trabajar en una propuesta evaluativa del sistema educativo que pudiera ayudar en la mejora y equidad del mismo. Para ello se optó por un sistema de evaluación basado en exámenes nacionales Sistema de Medición de la Calidad Educativa (SIMCE) en 1988. Lo que nació con una intencionalidad evaluativa de mejora, se ha transformado en un medio para detectar las escuelas con bajo rendimiento académico y estigmatizarlas; propio del criterio de competitividad de las políticas neoconservadoras del gobierno militar aún presentes, (CARNOY, 2010). El SIMCE y los exámenes de carácter internacional (TIMSS ${ }^{1}$, PISA $^{2}$ ) dan clara muestra de la dirección tomada por el país en lo que a educación se refiere.

\footnotetext{
${ }^{1}$ Third International Mathematics and Science. Estudio Internacional de Tendencias en Matemáticas y Ciencias.

${ }^{2}$ Programme for International Student Assessment. Programa Internacional para la Evaluación de Estudiantes.
} 
En el caso concreto de la Educación Física (EF), el Sistema de Medición de la Calidad (SIMCEEF) se aplica por primera vez en el año 2010, realizándose una segunda aplicación en el 2011 a una muestra representativa de estudiantes de $8^{\circ}$ básico (13-14 años). Si bien el SIMCE tiene como:

[... ] propósito principal contribuir al mejoramiento de la calidad y equidad de la educación, informando sobre el desempeño de los estudiantes en diferentes área de aprendizaje del Currículum Nacional, y relacionando estos desempeños con el contexto escolar y social en que aprenden $[\ldots]$ evaluando

[...] el logro de los Objetivos Fundamentales y Contenidos Mínimos Obligatorios del Marco Curricular vigente en diferentes sectores de aprendizaje. (SIMCE, 2011, p.1) en el área de la EF adquiere una lógica totalmente diferente, focalizándose exclusivamente, en

[...] diagnosticar la condición física de los estudiantes y establecer una línea de base para futuras evaluaciones. (SIMCEEF, 2011, p.3)

El SIMCEEF se aplica midiendo cuatro parámetros (Antropometría, Rendimiento muscular, Flexibilidad y Resistencia Cardiorespiratoria); analizando sucintamente los últimos resultados de la aplicación del SIMCEEF en 2011, muestran un mapa de la población escolar chilena con altos índices de sobrepeso y obesidad que pueden conllevar a padecer en un futuro enfermedades cardiacas y metabólicas. Pero al mismo tiempo, la mayoría de ellos presentan índices de resistencia muscular y cardio-respiratoria aceptables.

Debemos tener en cuenta que el currículum chileno para el área de EF, al menos sobre el papel, es eminentemente de carácter cerrado, siendo de obligado cumplimiento el desarrollo de las Unidades prescritas para cada uno de los niveles. En cada una de ellas se identifican, por este orden, los contenidos a desarrollar, los objetivos definidos en base a resultados esperados, las orientaciones didácticas, las actividades a realizar con diferentes ejemplos de cada una de 
ellas (opcionales) y los criterios de evaluación a tener en cuenta. Sólo se deja en manos del docente la concreción de las actividades a realizar en cada una de las clases, respetando la distribución temporal marcada inicialmente para cada una de las unidades que integran el nivel. Lo que llama poderosamente la atención es que para un currículum que se ofrece como equilibrado en cuanto a los objetivos y contenidos planteados, centra la evaluación del mismo en tan sólo un treinta por ciento del mismo, lo que corresponde al bloque "Actividad física saludable".

Frente al modelo de evaluación externa que acabamos de describir, la literatura existente relacionada con la calidad de la $\mathrm{EF}$, nos muestra cómo dicho concepto suele atender a una realidad educativa más compleja, amplia y enriquecedora que la representada por el SIMCEEF. Penny et. al. (2009) reconocen que la calidad de los programas de EF y salud es un elemento que va más allá de las horas oficiales dedicada a la asignatura, debiéndose considerar otros elementos como: horas de actividad física extracurriculares, formación del profesorado, los programas, los estudiantes, dinámicas pedagógicas, el aprendizaje co-construido, la investigación existente sobre prácticas saludables, comunidad donde se realiza la actividad física, entre otros aspectos importantes a considerar. Esta diversidad de elementos a considerar nos obligaría a modificar sustancialmente el perfil de las pruebas utilizadas para evaluar la calidad de la EF (MENEAR; SIMS; PHILLIPS 2007).

Incluso las propuestas evaluativas norteamericanas y australianas basadas en competencias cuentan con una perspectiva más amplia al incluir conocimientos teóricos y prácticos, actitudes y valores sociales, así como colaboraciones con agentes sociales diversos (AAHPERD, 2013; ALLIANCE..., 2013; NASPE, 2013).

Partiendo de lo expuesto y teniendo en cuenta los resultados descritos anteriormente de la última aplicación del SIMCEEF en 2011, nos planteamos como objetivo: analizar críticamente el Sistema de Medición de la Educación Física Chilena. Concretamente queremos describir el concepto de calidad de la EF en la que está centrada esta política evaluativa; desvelar las finalidades implícitas 
en este sistema de evaluación; identificar las concepciones evaluativas; analizar la relación entre el SIMCEEF y la desigualdad educativa presente en el país, e interpretar el rol del docente de EF construido a partir de este instrumento evaluativo.

\section{Metodología}

Hemos optado por una lógica investigativa anclada en el paradigma interpretativo-fenomenológico a partir del que creemos es posible inferir y comprender dichos significados (CANALES, 2006; RUIZ, 2003). Desde este enfoque paradigmático, la metodología cualitativa nos permite una comprensión subjetiva que emerge a partir de los documentos analizados, entendiendo que los mismos esconden un contenido educativo caracterizados, implícita y/o explícitamente, por intencionalidades educativas, finalidades, concepciones, exclusiones, valoraciones, etc... Entendemos que dichos documentos "son ejemplos de comunicación humana significativa" (GIBBS, 2012, p.28), utilizando para su descripción, interpretación y comprensión de cualidades los conceptos y relaciones existentes entre ellos (STRAUSS; CORBIN, 2002; GIBBS, 2012).

Los documentos analizados fueron los dos Informes de Resultados del SIMCE en EF (años 2010 y 2011), que se han constituido en el único material oficial existente para explicar qué es, para qué sirve, cómo se aplica y quien debe llevar a cabo la evaluación de la calidad de la EF chilena. No hay, a nivel ministerial y académico, otra información al respecto, por lo que hemos creído muy relevante hacer este análisis crítico. Complementariamente y con la finalidad de poder contrastar la información, se han analizado los currículum de $5^{\circ}, 6^{\circ}, 7^{\circ}$ y $8^{\circ}$ curso básico, concretamente la propuesta de objetivos y contenidos.

Los documentos han sido analizados con la ayuda del NVivo 9.0. Los procedimientos empleados, en general se ciñeron a las propuestas de fragmentación y articulación de la Grounded Theory (STRAUSS; CORBIN, 2002; GLASER; STRAUSS, 1967). Se realizó inicialmente una primera fase de análisis inductivo, aunque 
debemos reconocer que los objetivos han orientado en parte el proceso, en el que fueron apareciendo las categorías previas (nudos vivos) presentes en los documentos. Posteriormente, se procede a una organización axial bajo las metáforas principales de análisis (figura 1) siguiendo la propuesta de reducción de la información producida de Strauss y Corbin (2002, p. 110) y teniendo en cuenta "criterios temáticos" (RODRÍGUEZ; GIL; GARCÍA, 1999, p.207). En un segundo momento, de profundización y afinamiento (MILES; HUBERMAN, 1994), gracias a la elaboración de matrices, se han localizado las relaciones más significativas existentes entre las categorías, lo que ha permitido el planteamiento de micro-hipótesis (interrogantes surgidos de un análisis de carácter más deductivo), tal y como propone Gibbs (2012). Por último, las teorías sustantivas construidas a partir del análisis y expuestas en el informe y reflejadas en la figura 1, nos han permitido cerrar la investigación desde unas conclusiones finales referidas a los hallazgos más significados de la investigación (RUIZ, 2003; STRAUSS; CORBIN, 2002).

Figura 1: Relación entre las principales metáforas del análisis

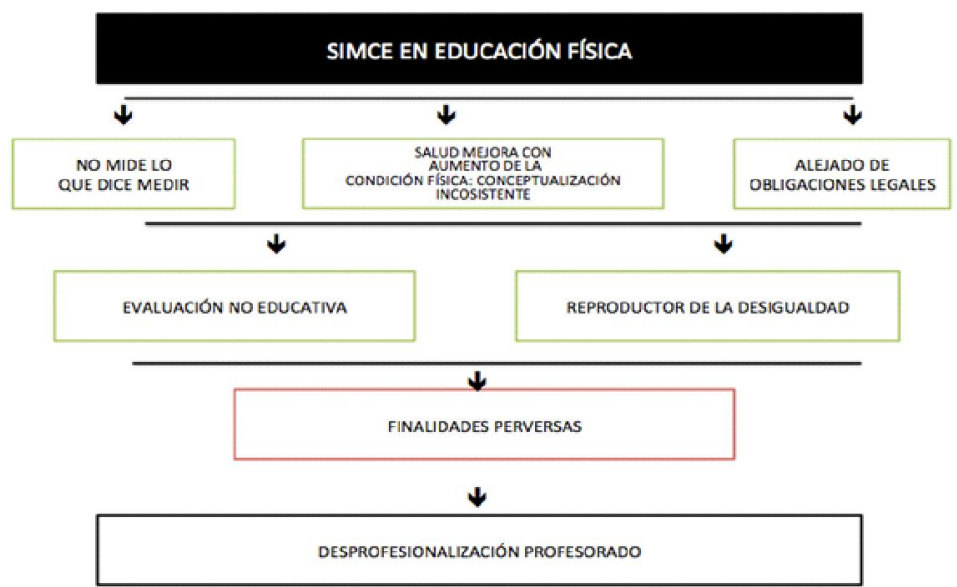

Fuente: elaboración propia de los autores. 


\section{Resultados y discusión}

\subsection{EL SIMCEEF NO MIDE LO QUE DICE MEDIR}

La primera idea que llama poderosamente la atención es la falta de coherencia entre el significado de las siglas SIMCE (Sistema de Medición de la Calidad de la Educación) y la lógica de la propuesta para el área curricular de la EF. Se referencia, textualmente, que "los resultados SIMCE son la principal herramienta de información del sistema educativo sobre los aprendizajes" [...] y "que en él se entregan los resultados de la [...] evaluación nacional de Educación Física [...] logrados por los estudiantes en los diferentes ciclos de enseñanza" (SIMCE 2011, p.1).

Como podemos observar en la tabla 1 , el porcentaje de presencia de cada uno de los bloques de contenidos de la EF escolar de $5^{\circ}$ a $8^{\circ}$ básico es prácticamente idéntico. Sin embargo, el SIMCEEF se focaliza en una forma muy especial de entender el bloque de contenido Actividad Física Saludable.

Tabla 1- Presencia de los bloques de contenidos de EF en cada uno de los cursos de $5^{\circ}$ a $8^{\circ}$ de la enseñanza básica.

\begin{tabular}{lcccc}
\hline Con tenidos & $\begin{array}{c}\text { Actividades } \\
\text { Físicas } \\
\text { Naturaleza }\end{array}$ & $\begin{array}{c}\text { Actividad } \\
\text { Física } \\
\text { Saludable }\end{array}$ & $\begin{array}{c}\text { Danza y } \\
\text { Expresión } \\
\text { Corporal }\end{array}$ & $\begin{array}{c}\text { Juegos y } \\
\text { Deportes }\end{array}$ \\
\hline $5^{\circ}$ & $25 \%$ & $50 \%$ & $0 \%$ & $25 \%$ \\
$6^{\circ}$ & $25 \%$ & $25 \%$ & $25 \%$ & $25 \%$ \\
$7^{\circ}$ & $25 \%$ & $25 \%$ & $25 \%$ & $25 \%$ \\
$8^{\circ}$ & $25 \%$ & $25 \%$ & $25 \%$ & $25 \%$ \\
\hline
\end{tabular}

Fuente: elaboración propia de los autores

En segundo lugar, si entendemos que la construcción de hábitos saludables depende, principalmente, del desarrollo de ciertas actitudes relacionadas con la práctica de actividad física, llama poderosamente la atención cómo en el análisis realizado del tipo de verbo presente en los objetivos del currículum para la EF, elaborado desde la categorización y posterior cruce de matriz con cada uno de los cursos y bloques realizado con el Nvivo, los objetivos se orientan al desarrollo de conceptos y procedimientos $(85,7 \%)$, obviando prácticamente el 
desarrollo de actitudes (Tabla 2). Pareciera entenderse que trabajando ciertos conceptos y practicando actividad física conseguiremos generar el hábito (actitudes) que como profesores de EF estamos buscando, lo que parece un error didáctico bastante importante. Más aun considerando la falta de horas destinadas a la EF escolar. Partiendo de que el SIMCEEF se centra en la evaluación del bloque Actividad Física Saludable, aún es más limitante, profesionalmente, al obviar los logros alcanzados por los estudiantes respectos a los objetivos conceptuales y actitudinales, los que representan un 57,1\% de los aprendizajes esperados. El SIMCEEF se centra, casi exclusivamente, en los objetivos de carácter procedimental.

Tabla 2 - Presencia de los tipos de contenido presente en la definición de los objetivos de $\mathrm{EF}$ en el currículum de $5^{\circ}$ a $8^{\circ}$ de la enseñanza básica.

\begin{tabular}{lccc}
\hline \multicolumn{1}{c}{ Tipo Contenido } & Actitudinales & Conceptuales & Procedi mentales \\
Contenidos & $20 \%$ & $0 \%$ & $80 \%$ \\
\hline Actividad Física Naturaleza & $14.3 \%$ & $42,9 \%$ & $42,9 \%$ \\
Actividad Física Saludable & $25 \%$ & $0 \%$ & $75 \%$ \\
Danza y Expresión Corporal & $42,9 \%$ & $14,3 \%$ & $42,9 \%$ \\
Juegos y Deportes & & \\
\hline
\end{tabular}

Fuente: elaboración propia de los autores

Resulta sorprendente pensar que el uso de un sistema evaluativo centrado en la condición física de los niños y niñas, nos pueda entregar información suficiente para diagnosticar la calidad de un área curricular cuyo eje vertebrador no está en dicha condición física sino como señala en propio Ministerio de Educación, el "desarrollo de una actitud crítica y analítica frente a las propias actividades físicas y a la comprensión de los cambios biológicos y psicológicos que los y las jóvenes están experimentando en esta etapa" (MINEDUC, 2010, p.5).

Es de sentido común, entonces, que asalten algunas preguntas: ¿cómo se está evaluando la actitud crítica y analítica propuesta?; ¿comprenden los estudiantes los cambios biológicos y psicológicos que están vivenciando?; ¿cómo el profesorado está abordando su labor pedagógica en la consecución de estas loables metas educativas?; ¿qué comprensión podemos construir a partir del SIMCEEF para contribuir a la construcción de hábitos de actividad 
física saludable entre la población escolar? La respuesta es clara y contundente: el SIMCEEF no es un instrumento para medir la calidad del área curricular en cuestión, sino un instrumento para medir nivel de condición física de los estudiantes.

Sin duda que la situación actual en relación a los altos índices de obesidad y sedentarismo, unidos a una condición física inadecuada de los niños y jóvenes, es una preocupación internacional (HARDMAN; MARSHALL, 2000; HARDMAN; MARSHALL, 2009), pero es ingenuo pensar que la calidad de la EF debe medirse a raíz de los resultados alcanzados en estas materias, como si fuera la asignatura la única responsable de tal situación.

Existe una negación absoluta del trabajo académico y científico que se viene desarrollando en los últimos años en relación a la construcción de indicadores que permitan identificar cuáles serían los criterios a partir de los cuales podemos definir la calidad de la EF. Unesco y North Western Counties Physical Education Association (2011) entienden que para llegar a tener una propuesta de este tipo es necesario trabajar en un modelo de necesidades básicas sobre EF de calidad, considerando aspectos tan diversos como: políticas nacionales; objetivos y contenidos; calidad de la impartición; supervisión; equidad; recursos humanos y materiales; condiciones profesionales; concepciones en relación al concepto de calidad de la EF; ejemplos existentes de prácticas consideradas idóneas. Algo similar nos proponen Penney et al. (2009) y Castejón $(2013)^{3}$.

La complejidad de una expresión polisémica como "calidad de la educación física" es simplificada y distorsionada en el SIMCEEF a través del concepto de condición física. Como diría Santos (2003, p.21) "simplificada abusivamente", al identificar el éxito con el rendimiento biofisiológico ${ }^{4}$ del alumno y por verificar el rendimiento

\footnotetext{
${ }^{3}$ Lógicamente hay diferencias entre las tres propuestas referenciadas, pero mantienen algo en común: atender a los elementos cualitativos de la práctica docente-discente en la educación física escolar.

${ }^{4}$ Santos Guerra utiliza esta caracterización para hablar de la escuela en general, no sólo de la educación física. Nosotros hemos creído importante contextualizar sus palabras al área de estudio en el que estamos implicados.
} 
a través de pruebas poco significativas y contradictorias con el currículum propuesto.

\subsection{RELACIÓN SALUD-CONDICIÓN FÍSICA: INCOSISTENCIAS TEÓRICAS}

Desde el propio informe se asume que el conocimiento de los niveles de condición física nos llevará a tener información para construir propuestas educativas promotoras de estilos de vida saludable. "Los resultados de esta evaluación permiten [...] elaborar planes de acción destinados a promover la actividad física y un estilo de vida saludable en los estudiantes" (SIMCEEF, 2011, p.7). Pero lo realmente grave es la relación causa y efecto entre resultados negativos, escasa realización de actividad física y poseer estilos de vida poco saludables. En definitiva, se asume implícitamente la relación calidad pedagógica de la EF con alto nivel de condición física.

La evidencia científica nos señala que es arriesgado centrar los esfuerzos de mejora de la salud en el aumento de los niveles de condición física, sobre todo si hablamos de niños y jóvenes, pues en esas edades los niveles están determinados por factores genéticos y de maduración, y otros aspectos sociales y medioambientales (BOUCHARD; SHEPARD, 1993); más que por hábitos de actividad física (KATZMARZYK et. al,. 1998). Por el contrario, sí hay consenso en que la práctica regular de actividad física se relaciona con un mejor bienestar personal y social. ¿Por qué no evaluar, entonces, la autonomía construida en las clases de EF en relación a la autogestión de un propio plan de trabajo de actividad física relacionada con la salud? ¿Cómo se provoca, desde la práctica pedagógica escolar, la generación de un hábito de práctica de actividad física?

Si nos centramos en aumentar los niveles de condición física sin atender a aspectos como la competencia percibida, obesidad y las burlas de los compañeros, demandas sociales y preferencias personales, la familia, zonas donde se vive, etc., podríamos estar afectando identidades inactivas durante la adolescencia (DEVÍS; BELTRÁN; PEIRÓ, 2013). Entendemos que el esfuerzo debe 
centrarse en generar hábitos, frente a mantener y conseguir una adecuada condición física en los estudiantes (BLAIR; CHENG; HOLDER, 2001).

\subsection{INADECUADO CUMPLIMIENTO DE LA LEY DEL DEPORTE}

Si bien este es un aspecto muy puntual del análisis crítico realizado, hemos querido mostrarlo por la importancia que tiene establecer una política coherente con la legislación vigente. En la Ley del Deporte chilena (2005) se establece que "El Ministerio de Educación establecerá un Sistema Nacional de Medición de la Calidad de la Educación Física y Deportiva para ser aplicado al finalizar la Educación Básica, debiendo consultar previamente al Instituto Nacional de Deportes de Chile" (CHILE, 2005). El SIMCEEF, tal y como está quedando en evidencia, no mide la calidad de la EF, simplemente porque se centra en la valoración del nivel de condición física de los escolares, sin entrar en la evaluación de aspectos educativos, tales como "impulsar en ellos (los estudiantes) una conducta crítica y analítica frente a sus propias actividades físicas" (MINEDUC, 2010, p.5) que trascienden los aspectos biofisiológicos propios del nivel de condición física, eje central de la evaluación practicada.

\subsection{EVALUACIÓN NO EDUCATIVA Y REPRODUCTORA DE LA DESIGUALDAD EXISTENTE}

Entendemos que una evaluación educativa, siguiendo a Santos (1995), es un proceso global (evalúa a todos los actores) y autocontrolado. Su objetivo será la mejora de la práctica y de las personas que participan de ella, siendo respetuoso con las mismas.

En el SIMCEEF, la evaluación está centrada, de forma exclusiva, en los alumnos que intervienen, sin atender al profesorado, la calidad de las instalaciones, el establecimiento educacional, al realidad social de los niños $/ \mathrm{as}^{5}$, etc..."El objetivo es diagnosticar el

\footnotetext{
${ }^{5}$ Aunque sí se harán análisis al respecto para decir que los niños de colegios municipales son los que peores resultados obtienen. Pero dichos análisis no aportan en la comprensión de estos resultados en función de la realidad social, cultural y económica.
} 
estado de la condición física de los estudiantes" (SIMCEEF, 2011, p.3)

Nos sorprendente que desde las políticas públicas se proponga un sistema evaluativo centrado, exclusivamente, en la condición física de los niños y niñas, y que se crea que nos pueda entregar información suficiente para establecer directrices pedagógicas para la EF escolar que permitan abordar, educativamente, los problemas existentes. El SIMCEEF es una clara muestra de que en la EF aún siguen muy presentes las concepciones evaluativas centradas en una visión tradicional del área. Pensando que son las estrategias reproductoras de un saber objetivo, observable y medible (rendimiento motor) las que deben representar la mayor parte del trabajo pedagógico (LÓPEZ , 2004).

Las desigualdades sociales y educativas, ya enunciadas, se focalizan principalmente en una redistribución del ingreso poco equitativa, un sistema educativo muy segregado por clases sociales, muy desigual en función de la cercanía o lejanía de la capital, ingresos económicos dispares para hombres y mujeres y con poca atención a las minorías (CARNOY 2010; MAYOL, 2012; VILLALOBOS; VALENZUELA, 2012). Este sistema de medición de la calidad reproduce algunas de esas desigualdades: diferentes niveles de condición física en función del sexo, nivel socioeconómico o tipo de establecimiento educacional al que los alumnos asisten. Estas diferencias sólo se usan para constatar que niños y niñas de diferente sectores poseen diferentes resultados, pero no para contextualizar la evaluación y aportar en la comprensión de la realidad. En el apartado siguiente daremos cuenta de la perversidad que esto supone.

Ante la escasez de información que produce el SIMCEEF, es complejo comprender el estado actual de la asignatura. El foco: niveles de condición física de los estudiantes, es sólo una pequeña parte de ella, ni siquiera la más importante (MORENO, 2011). Ante esta realidad las orientaciones pedagógicas que el SIMCEEF nos estrega son generales, por lo que no se entiende el aparataje evaluativo generado. Veamos lo que dice el informe al respecto (SIMCEEF, 2011, p.50): 
De acuerdo a los programas de estudio del sector y del nivel, para la elaboración de los planes de trabajo individuales, se debe considerar lo siguiente:

"Las actividades deben ser trabajadas a lo largo del año. En este sentido, durante la organización de las clases, se debe prever un tiempo específico para llevar a cabo actividades inherentes a este eje de contenido, sin descuidar los otros contenidos curriculares.

"Los planes de trabajo se tienen que ejecutar dentro y fuera delámbito escolar. Para ello, se deben diseñar considerando las posibilidades de aplicación tanto dentro como fuera del establecimiento. Como herramienta de automonitoreo, se recomienda que los estudiantes construyan una bitácora de trabajo semanal, en que anoten y describan los ejercicios y las actividades realizadas de manera sistemática.

"Los planes de trabajo deben incluir objetivos y metas, tipos de ejercicios a realizar, cantidad de sesiones semanales y hábitos de vida saludable.

"Se debe determinar qué evaluaciones se utilizarán periódicamente para que los estudiantes evalúen sus progresos personales en función de los compromisos adquiridos.

Lo planteado puede ayudar a mejorar los niveles de condición física de los estudiantes, pero es una propuesta excesivamente específica para ser una propuesta que ayude a mejorar, realmente, las prácticas de la EF escolar.

\subsection{FINALIDADES PERVERSAS DEL SIMCEEF Y DESPROFESIONALIZACIÓN DEL PROFESORAdO dE EdUCACIÓN FísICA}

Vamos a detenernos seguidamente en las finalidades más significativas explicitadas por el SIMCE y su utilidad para profesores y directivos y de cómo estas pueden estar perjudicando y tergiversando el proceso educativo en la EF Escolar.

"Evaluar si las iniciativas implementadas en la escuela tuvieron un efecto positivo en el nivel de desempeño de los estudiantes" 
(AGENCIA DE CALIDAD DE LA EDUCACIÓN, 2013, p.1). Se asume, implícitamente, que los establecimientos educacionales y los profesores de EF están tomando medidas para solventar el gran problema que el SIMCEEF señala: falta de condición física adecuada. ¿Por qué esa problemática y no otras? Por ejemplo las carencias comunicativas, de autonomía y autoestima, que presentan los estudiantes y que se podrían trabajar desde otros contenidos del currículum como la Expresión Corporal o los Juegos Deportivos. Lo que si queda claro es que el SIMCEEF está condicionando la práctica real de los docentes. Un buen ejemplo de ello lo podemos encontrar en esta cita recogida en Moreno et. al. (2013, p.13): "Me pusieron dos horas extras, o sea, ese curso tenía cuatro horas para la preparación del SIMCEEF [...]. En esas cuatro horas los chicos terminaron colapsados con acondicionamiento físico" (Soledad).

Efectivamente, algunos establecimientos educacionales comienzan a ponerse al servicio del sistema de evaluación, perdiendo la necesaria acción crítica y el compromiso con los que aprenden (ÁLVAREZ, 2006). Como señalaba otro docente (Gabriel) de la investigación anterior, "[... ] te van hacer trabajar para el SIMCEEF" (MORENO et. al. 2013, p.13)

Para el SIMCE, las evaluaciones realizadas podrían servir, a los padres, para "[...] conocer cómo le fue a su escuela en comparación con otras escuelas de la misma comuna o dependencia." Instaurar el "semáforo escolar", en relación a la construcción de un mapa de centros que señale los diferentes niveles de calidad. Se asume, de forma perversa, que aquellos centros educativos y aquellas clases de EF con menores rendimientos son resultados de un trabajo pedagógico de menor calidad. Quien fuera Ministro de Educación durante la aplicación del primer SIMCEEF, tras los resultados en el mismo, señaló que "[...] las actuales horas de Educación Física se están haciendo muy mal" (COOPERATIVA, 2010, p.1). Se relaciona profesionalidad docente y calidad de la EF con nivel de condición física alcanzado por los estudiantes (dar respuesta acrítica a lo que Ministerio propone); nunca con una práctica docente educativa, caracterizada por un accionar crítico contextualizado (FREIRE, 
2005), democrático, participativo y comunitario (STEINBERG; FLECHA, 2009) en el quehacer profesional.

$\mathrm{Al}$ propio Ministerio, el SIMCE le servirá para monitorear la calidad y equidad de la educación desde la perspectiva de los logros de aprendizaje que alcanzan los estudiantes en distintas áreas curriculares (AGENCIA DE CALIDAD DE LA EDUCACIÓN, 2013). ¿De qué sirve constatar la inequidad del sistema educativo, ya conocida además, si no se va a proponer una prática de la EF escolar que propenda a la compensación de las mismas? (DEVÍS; MOLINA 2004; MORENO 2011). Como también señala Diniz, Franco y Silvia (2009), se sigue incentivando una EF centrada en una mirada tecnocrática del proceso educativo y en una evaluación que sirve más para constatar niveles de rendimiento que para comprender procesos educativos.

\section{CONCLUSIONES}

El SIMCEEF no da respuesta a un concepto de calidad previamente explicitado. Ni siquiera entrega información de cómo en la EF se está consiguiendo, o no, desarrollar los objetivos curriculares explicitados en los planes y programas existentes para la asignatura.

La relación conceptual que se establece entre salud y condición física responde a una limitada perspectiva teórica en la que se entiende que a mayor condición física mayor y mejor bienestar humano. Esta es una relación muy arriesgada, sobre todo en el grupo de niños que participan de este sistema de medición.

Es un sistema de evaluación que tiende a reproducir y constatar la desigualdad entre los diferentes sectores sociales presente en el país y, por tanto, no se constituye en una lógica evaluativa que permita transformar el status quo imperante.

El SIMCEEF pretende tener valor en sí mismo y no ser un medio para mejorar la práctica educativa. Ello tiene el riesgo de 
generar una labor docente más centrada en dar respuesta al propio instrumento que en ser un aporte para la generación de hábitos saludables de actividad física a partir de las prácticas educativas. 


\begin{abstract}
Chilean Physical Education quality measurement system: a critical analysis

Abstract: This article analyses, qualitatively and critically, the assessment system used by the Chilean Education Ministry for the quality measurement of Physical Education as a subject in the school curriculum. Generally, the analysis procedures used follow the fragmentation and articulation proposals from the "Grounded Theory". The conclusion is that such a system does not answer to a quality concept previously specified. It shows an important conceptual limitation in the health/physical condition relationship. It establishes an assessment logic that is very limited as far as training purposes are concerned and at the same time generates some professional underrating for the teachers.
\end{abstract}

Key words: quality management. Curriculum. Measures.

\begin{abstract}
Sistema de medição de qualidade da Educação Física chilena: análise crítica

Resumo: Este trabalho analisa, de maneira qualitativa e crítica, o sistema de avaliação utilizado pelo Ministério de Educação do Chile para medir a qualidade da educação física como matéria do currículo escolar. Em geral, os procedimentos de análises utilizados se basearam nas propostas de fragmentação e articulação da "Grounded Theory". Concluímos que este sistema não responde ao conceito de qualidade explícito previamente; mostra uma limitação conceitual importante na relação da saúde e da condição física; estabelece uma lógica de avaliação escassamente formativa e gera certa desvalorização profissional dos docentes.
\end{abstract}

Palavras-Chave: Gestão de qualidade. Currículo. Medidas. 


\section{REFERENCIAS}

AGENCIA DE CALIDAD DE LA EDUCACIÓN. ¿Qué es el SIMCE? Santiago de Chile: Gobierno de Chile. Disponible en: <http://www.agenciaeducacion.cl/simce/quees-el-simce/>. Acceso en: 5 sep. 2013.

ALLIANCE FOR A HELTHIER GENERATION. Let's Move Active Schools. Disponible en: <https://www.healthiergeneration.org/news_events/2013/04/05/769/ lets_move_active_schools>. Acceso en: 9 jul. 2013.

ÁLVAREZ, Juan Manuel. Evaluar para conocer, examinar para excluir. Madrid: Morata. 2006.

AMERICAN ALLIANCE FOR HELTH, PHYSICAL EDUCATION, RECREATION AND DANCE. National Standards \& Grade-Level Outcomes for K-12 Physical Education. Disponible en: <http://www.aahperd.org/whatwedo/ nationalStandards.cfm> Acceso en: 9 jul. 2013.

BLAIR, Steven; CHENG, Yiling; HOLDER, Scott. Is physical activity or physical fitness more important in defining health benefits? Medicine and science in sports and exercise, Indianápolis, v. 33, 6, p. 379-399, 2001.

BOUCHARD, Claude; SHEPHARD, Roy. Physical activity, fitness and health: The model and key concepts. En: . BOUCHARD, R; SHEPHARD, R. Physical activity, fitness, and health: Consensus Statement. Champaign, IL: Human Kinetics, 1993. p. 11-20.

CANALES, Manuel. Metodologías de Investigación Social: Introducción a los oficios. Santiago de Chile: LOM, 2006.

CARNOY, Martin. La ventaja académica de Cuba. ¿Por qué los estudiantes cubanos rinden más? México: Fondo de Cultura Económica. 2010.

CASTEJÓN, Francisco. Indicadores de calidad en la enseñanza de la educación física. En: Revista Tándem Didáctica de la Educación Física, Barcelona, n. 41, p. 68-76, 2013.

CHILE. Presidente de la República. Ley del Deporte número 20019 de 5 de mayo de 2005. Regula las sociedades anónimas deportivas profesionales. Diário Oficial República de Chile, Santiago, 7 mayo 2005. Disponible en: < http://www.leychile.cl/ Navegar?idLey=20019\&idVersion=2005-05-07>. Acceso en: 13 dic. 2010.

COOPERATIVA SIMCE. Simce de Educación Física reveló que cuatro de cada 10 niños tiene sobrepeso. Disponible en <http://www.cooperativa.cl/simcede-educacion-fisica-revelo-que-cuatro-de-cada-10-ninos-tiene-sobrepeso/ prontus_nots/2011-03-09/122818.html> Acceso en: 13 dic. 2010.

DEVÍS, José; BELTRÁN, Vicente; PEIRÓ, Carmen. Exploring socio-ecological factors influencing activeand inactive Spanish students in years 12 and 13 . Sport, Education and Society, London, DOI:10.1080/13573322.2012.754753, 2013. 
DEVÍS, José; MOLINA, Joan. Las funciones de la educación física escolar: de la modernidad a la postmodernidad. En: CAPARRÓZ, A; ANDRADE, N. Investigação e intervenção. Vitória: UFES, 2004. p. 35-49.

DINIZ, Josiane; FRANCO, Amaral; SILVIA, Cristina. A Avaliação na Educação Física Escolar: Uma Comparação entre as Escolas Tradicional e Ciclada. Movimento, Porto Alegre, v. 15, n. 1, p. 241-258, 2009.

DONOSO, Sebastián; ARIAS, Óscar. Distribución desigual de las oportunidades educativas en el territorio y migración de la matrícula escolar: el caso de la región de Los Lagos (Chile). Revista Estudios Pedagógicos, Valdivia, v. 38, n. 2, p. 35-54, 2012.

ESCUDERO, Juan. Evaluación de las políticas educativas: cuestiones perennes y retos actuales. Revista Fuentes, Sevilla, n. 10, p. 8-21, 2010.

FREIRE, Paulo. La educación como práctica de libertad. Madrid: Siglo XXI, 2005.

GIBBS, Graham. El análisis de datos cualitativos en investigación cualitativa. Madrid: Morata, 2012.

GLASER, Barney; STRAUSS, Anselm. The discovery of grounded theory: Strategies for qualitative research. Chicago: Aldine, 1967.

GOLDSTEIN, H. Using pupil performance data for judging schools and teachers: Scope and limitation. British Educational Research Journal, London, v. 27, p. 433-442, 2001.

HARDMAN, Ken; MARSHALL, Joe. Second World-wide Survey of School Physical Education: Final Report. Berlin: ICSSPE/CIEPSS, 2009.

HARDMAN, Ken; MARSHALL, Joe. World-wide survey of the state and status of school physical education: Final Report. Manchester: University of Manchester, 2000.

KATZMARZYK, P.; et. al. Somatotype and indicators of metabolic fitness in youth. American Journal of Human Biology, Massachusetts, n. 10, p. 342-350, 1998.

LÓPEZ, Ramón; FIGUEROA, Eugenio; GUTIÉRREZ, Pablo. La 'parte del león': Nuevas estimaciones de la participación de los súper ricos en el ingreso de Chile. En: Serie de Documentos de Trabajo. Santiago de Chile: Universidad de Chile. Disponible en: <http://www.econ.uchile.cl/descargar/publicacion/la-parte-del-leon-nuevasestimaciones-de-la-participacion-de-los-super-ricos-en-el-ingreso-de-chile $>$. Acceso en: 9 jul. 2013.

LÓPEZ, Victor Manuel. La participación del alumnado en los procesos evaluativos: la autoevaluación y la evaluación compartida en Educación Física. En: FRAILE, A. Didáctica de la Educación Física: una perspectiva crítica y transversal. Madrid: Biblioteca Nueva, 2004. p. 265-290. 
MARTÍNEZ, Felipe. La evaluación de la calidad de los sistemas educativos: propuesta de un modelo. En: MARTÍN, E.; MARTÍNEZ, F. Avances y desafíos en la evaluación educativa. Madrid: Organización de Estados Iberoamericanos, 2011. p. 27-39.

MAYOL, Alberto. El derrumbe del modelo: la crisis de la economía de mercado en el Chile contemporáneo. Santiago de Chile: LOM Ediciones, 2012.

MENEAR, Kristi; SIMS, Sandra; PHILLIPS, John. Strategies Fitness Testing of Students with Disabilities: Comparing and Modifiying fitnes test to provide quality assesment for all students. En: Strategies, London, v. 20, n. 3, p. 12, 2007.

MILES, Michael; HUBERMAN, Matthew. Qualitative data analysis: an expanded sorcebook. Newbury Park: Sage. 1994.

MINEDUC. Ministerio de Educación. Planes y programas de estudio. Santiago de Chile. Disponible en: <www.mineduc.cl>. Acceso en: 9 jul. 2010.

MONARCA, Héctor. La racionalidad de las políticas de evaluación de la calidad de la educación. Revista Iberoamericana de Educación / Revista Iberoamericana de Educação Madrid, v. 59, n.1, p. 1-9, 2012.

MORENO, Alberto. et. al. La educación física chilena: un modelo tecnocrático de la enseñanza y desvalorización del colectivo docente. En: Tándem. Didáctica de la Educación Física, Barcelona, n. 42, p. 7-17, 2013.

MORENO, Alberto. Percepciones del profesorado universitario en relación a la función de transformación de la educación física como asignatura de curriculum escolar: el caso de Chile.2011. Tesis (Doctoral) - Granada: Universidad de Granada, 2011.

NASPE. National Standars and Grade-Level Outcomes for K-12 Physical Education. Disponible en: <http://www.aahperd.org/naspe/standards/ nationalStandards/PEstandards.cfm>. Acceso en: 09 jul. 2013.

OCDE. Definition and Selection of Competencies (DeSeCo): Theoretical and Conceptual Foundations: Strategy Paper. Disponible en:< http:// www.statistik.admin.ch/stat_ch/ber15/deseco/deseco_strategy_paper_final.pdf.>. Acceso en: 09 jul. 2013

OCDE. Economic Policy Reforms 2013: Going for Growth. París: OEDC . Disponible en: <http://dx.doi.org/10.1787/growth-2013-3n>. Acceso en: 09 jul. 2013.

PENNEY, Dawn; et. al. Curriculum, pedagogy and assessment: three message systems of schooling and dimensions of quality physical education. Sport, Education and Society, London, v. 14, 4, p. 421-442, 2009.

PÉREZ-GÓMEZ, José.; SOTO, Encarnación. Sentido educativo de las evaluaciones externas. Luces y sombras de PISA. Cultural y Educación, Barcelona, v.23, n.2, p. 171-182, 2011. 
PERRENOUD, Philipee. La evaluación de los alumnos: de la producción de la excelencia a la regulación de los aprendizajes. Entre dos lógicas. Buenos Aires: Colihue, 2008.

PUGA, Ismael. Escuela y estratificación social en Chile: ¿cuál es el rol de la municipalización y la educación particular subvencionada en la reproducción de la desigualdad social? En: Revista Estudios Pedagógicos, Valdivia, v. 37, n. 2, p. 213-232, 2011.

RODRÍGUEZ, Gregorio; GIL, Javier; GARCÍA, Eduardo. Metodología de la investigación cualitativa. Málaga: Aljibe, 1999.

RUIZ, José Ignacio. Metodología de la investigación cualitativa. Bilbao: Universidad de Deusto, 2003.

SANTOS, Miguel Ángel. La evaluación: un proceso de diálogo, comprensión y mejora. Málaga: Aljibe. 1995.

SANTOS, Miguel Ángel. Trampas en Educación: el discurso de la calidad. Barcelona: La Muralla. 2003.

SERVÁN, María. La evaluación externa de los aprendizajes escolares. Introducción. Cultura y Educación, Barcelona, v.23, n.2, p. 165-169, 2011.

SIMCE. Resultados SIMCE 2011: 4ํy y 8ํásico. Santiago de Chile: Ministerio de Educación, 2011.

SIMCEEF. Informe de Resultados Educación Física. Santiago de Chile: Ministerio de Educación. 2010.

SIMCEEF. Informe de Resultados Educación Física. Santiago de Chile: Ministerio de Educación. 2011.

SIMONS, Helen. School self-evaluation for development: The case for and reflections on a training programme. Cultura y Educación, Barcelona, v. 23, n.2, p 235-249, 2011.

STAKE, Robert. Evaluación comprensiva y evaluación basada en estándares. Barcelona: GRAÓ, 2006.

STEINBERG, Shirley; FLECHA, Ramón. Monográfico Pedagogía Crítica del Siglo XXI. Revista Electrónica Teoría de la Educación, Salamanca, v. 10, n. 3, p. 1-300, 2009.

STRAUSS, Anselm; CORBIN, Juliet. Bases de la investigación cualitativa: Técnicas y procedimientos para desarrollar la teoría fundamentada. Colombia: Universidad de Antioquia, 2002.

UNESCO. Project on the Development of Quality Physical Education: Indicators and Basic Needs Model. París: Disponible en: <http://www.unesco.org/new/ 
fileadmin/MULTIMEDIA/HQ/SHS/pdf/nwcpea_unesco_survey.pdf>. Acceso en: 09 jul. 2013.

VILLALOBOS, Cristobal; VALENZUELA, Juan Pablo. Polarización y cohesión social del sistema escolar chileno. Revista de Análisis Económico, Geogetown, v. 27, n. 2, p. 145-172, 2012.

Este artículo se ha realizado en el contexto y gracias al Proyecto de Investigación Fondecyt 11110016, titulado "La Educación Física y su función de transformación de las desigualdades sociales: profesorado del área y documentación ministerial", financiado por el Fondo Nacional de Investigación Científica y Tecnológica de Chile.

Dirección postal:

Pontificia Universidad Católica de Valparaíso

Facultad de Filosofía y Educación

Escuela de Educación

Grupo de Estudio "Motricidad y Educación"

Avenida El Bosque, 1290

Santa Inés

Viña del Mar

V Región

Chile

Recebido em: 30-07-2013

Aprovado em: 04-11-2013

Vovimento, Porto Alegre, v. 20, n. 01, p. 145-167, jan/mar de 2014. 